\title{
Exact ground state of a spin ladder with a quantum phase transition
}

\author{
W. Brzezicki ${ }^{1}$ and A.M. Oleśs ${ }^{1,2, a}$ \\ 1 Marian Smoluchowski Institute of Physics, Jagellonian University, Reymonta 4, 30059 Kraków, Poland \\ 2 Max-Planck-Institut für Festkörperforschung, Heisenbergstrasse 1, 70569 Stuttgart, Germany
}

Received 1st August 2008 / Received in final form 28 October 2008

Published online 4 December 2008 - (c) EDP Sciences, Società Italiana di Fisica, Springer-Verlag 2008

\begin{abstract}
We introduce a spin ladder with Ising interactions along the legs and intrinsically frustrated Heisenberg-like ferromagnetic interactions on the rungs. The model is solved exactly in the subspaces relevant for the ground state by mapping to the quantum Ising model, and we show that a first order quantum phase transition separates the classical from quantum regime, with the spin correlations on the rungs being either ferromagnetic or antiferromagnetic, and different spin excitations in both regimes. The present case resembles the quantum phase transition found in the compass model in one and two dimensions.
\end{abstract}

PACS. 75.10.Jm Quantized spin models - 64.70.Tg Quantum phase transitions - 75.10.Pq Spin chain models

\section{Introduction}

Spin ladders play an important role in quantum magnetism. Interest in them over the last two decades is motivated by their numerous experimental realizations in transition metal oxides [1]. One of them are spin ladders in $\mathrm{Sr}_{n-1} \mathrm{Cu}_{n+1} \mathrm{O}_{2 n}$ cuprates (with $n=3,5,7, \cdots$ ) [2], and the simplest of them, a spin ladder with two legs connected by rungs, is realized in $\mathrm{Sr}_{2} \mathrm{Cu}_{4} \mathrm{O}_{6}$. Another example of an antiferromagnetic (AFM) spin ladder with two legs and charge order is found in $\alpha^{\prime}-\mathrm{NaV}_{2} \mathrm{O}_{5}$ [3]. Excitation spectra of such AFM spin ladders are rich and were understood only in the last decade. They consist of triplet excitation, bound states and two-particle continuum [4], and were calculated in unprecedented detail for quantum AFM spin 1/2 two-leg ladder employing optimally chosen unitary transformation [5]. This advance in the theoretical understanding of the excitation spectra of spin ladders is accompanied by recent experimental investigations of one- and two-triplon spectra of another almost perfect spin-ladder compound $\mathrm{La}_{4} \mathrm{Sr}_{10} \mathrm{Cu}_{24} \mathrm{O}_{41}$ by inelastic neutron scattering [6]. Finally, in the theory spin ladders could serve as a testing ground for new (ordered or disordered) phases which might arise for various frustrated exchange interactions [7].

An interesting situation arises when spin interactions are frustrated and could thus lead to a disordered phase. While periodically distributed frustrated Ising interac-

\footnotetext{
a e-mail: a.m.oles@fkf.mpg.de
}

tions do not destroy magnetic long-range order which still occurs at low temperature below a magnetic phase transition [8], frustrated interactions in one-dimensional (1D) models could only lead to quantum phase transitions [9]. Frustrated interactions occur for a spin ladder in a natural way in the orbital part of the superexchange in spinorbital models, where frustration of the orbital part of the superexchange is intrinsic [10], and leads to disordered ground states. Examples of this behavior are either various valence-bond phases [11], or orbital liquids [12], while enhanced quantum effects and entanglement [13] are prominent manifestations of the quantum nature of such interactions. Also in doped systems some unexpected features emerge for frustrated orbital superexchange interactions [14]. Therefore, it is of great interest to investigate spin models with frustrated interactions, particularly when they can be exactly solved.

The orbital interactions are frequently Ising-like but different spin components interact depending on the bond orientation in real space [15], which generates frustrated interactions in the so-called two-dimensional (2D) quantum compass model. Recently this model was investigated numerically [16-18], while its $1 \mathrm{D}$ variant was solved exactly by an analytic method [9]. The latter model is equivalent to the 1D anisotropic XY model [19] in the limit of equal and alternating interactions on the bonds. In both $1 \mathrm{D}$ and 2D compass model one finds a highly degenerate disordered ground state, while small anisotropy of interactions lifts the degeneracy and leads to particular shortrange correlations dictated by the stronger interaction. An 
exact solution of the $1 \mathrm{D}$ compass model demonstrates that the nearest-neighbor spin correlation functions change discontinuously at a quantum phase transition (QPT) - a similar behavior was also found numerically for the $2 \mathrm{D}$ model [16].

The purpose of this paper is to present an exact solution of a spin ladder with frustrated interactions and a first order QPT between two regimes characterized by different spin correlations. We consider interactions on the rungs which interpolate between the Ising-like and the ones in the quantum $X Y$ model. Although these interactions do not correspond to any realistic situation, they stand for a generic competition between Ising and Heisenberg-like interactions in spin models which are likely to play a role in the orbital physics, and exhibit certain similarity to the compass model $[16,17]$. We show that the transition between two different ground states which occurs for an isolated rung survives in a spin ladder and dominates its behavior.

The paper is organized as follows. The model and its invariant dimer subspaces are introduced in Section 2. The ground state of the model is found in Section 3 by solving the model in two relevant subspaces, the one equivalent to the 1D quantum Ising model (QIM) and the classical one. Using an example of a finite system, we provide a justification that the ground state may belong only to one of these two subspaces. We also present the changes of spin correlations at the QPT, which are derived from the respective ground state energies. In Section 4 a brief discussion of spin excitations in the vicinity of the QPT is presented. Final discussion and summary of the results are given in Section 5 .

\section{Frustrated spin ladder}

\subsection{The model and frustration of interactions}

We consider a spin ladder with $2 N$ spins $s=1 / 2-$ two spin chains are joined by $\langle 2 i-1,2 i\rangle$ rungs, as shown in Figure 1. It is straightforward to realize that when the interactions on the rungs are ferromagnetic (FM) and vary between the Ising and the XY limit, one finds a competition between the local triplet states on each rung: $\{|\uparrow \uparrow\rangle,|\downarrow \downarrow\rangle\}$ and $(|\uparrow \downarrow\rangle-|\downarrow \uparrow\rangle) / \sqrt{2}$. Here $\{|\uparrow\rangle,|\downarrow\rangle\}$ are $s_{i}=1,-1$ eigenstates of $\sigma_{i}^{z}$ Pauli matrix. We show that the transition between the two above ground states in an isolated rung survives in a spin ladder with AFM Ising interactions along the legs [20],

$$
\begin{aligned}
\mathcal{H}(\alpha, \beta)= & J \sum_{i=1}^{N}\left\{-\alpha\left(\sigma_{2 i-1}^{x} \sigma_{2 i}^{x}+\sigma_{2 i-1}^{y} \sigma_{2 i}^{y}\right)\right. \\
& \left.-(1-\alpha) \sigma_{2 i-1}^{z} \sigma_{2 i}^{z}\right\} \\
& +J \beta \sum_{i=1}^{N}\left(\sigma_{2 i-1}^{z} \sigma_{2 i+1}^{z}+\sigma_{2 i}^{z} \sigma_{2 i+2}^{z}\right)
\end{aligned}
$$

where $\left\{\sigma_{i}^{x}, \sigma_{i}^{y}, \sigma_{i}^{z}\right\}$ are the Pauli matrices, and we use periodic boundary conditions: $\sigma_{2 N+1}^{z} \equiv \sigma_{1}^{z}, \sigma_{2 N+2}^{z} \equiv \sigma_{2}^{z}$. For simplicity we assume that $N$ is even.

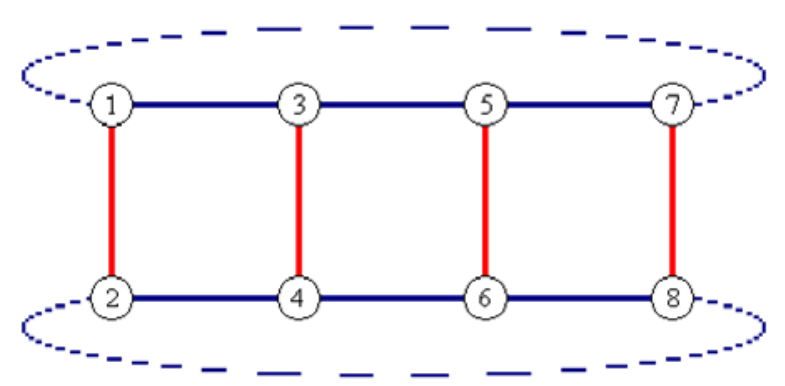

Fig. 1. Schematic view of the ladder with the convention of site labels used in the text: each rung $i$ connects the sites $2 i-1$ and $2 i$. Ising interactions along the ladder legs (1D chains) and Heisenberg-like ones along the rungs are given in equation (1). Dashed lines indicate the periodic boundary condition for the present ladder with $N=4$ rungs.

The spin ladder model (1) depends on two parameters: $0 \leq \alpha \leq 1$ and $\beta \geq 0$. The interactions on the rungs are Heisenberg-like with all three spin components, coupled by two FM interactions: $\alpha J$ for $x$-th and $y$-th spin components and $(1-\alpha) J$ for $z$-th ones, and the rungs are coupled by the AF Ising interactions $\beta J$, so it is convenient to use the following parameters:

$$
J_{1} \equiv \alpha J, \quad J_{2} \equiv(1-\alpha) J, \quad K \equiv \beta J .
$$

We will use the basis of vectors $\left\{\left|s_{1}, s_{2}, \cdots, s_{2 N}\right\rangle\right\}$ defined by the eigenstates of $\sigma_{i}^{z}$ operators at each site $i$ (with eigenvalues $s_{i}= \pm 1$ ) to introduce the relevant subspaces.

It is straightforward to see that the XY quantum interaction $\propto\left(\sigma_{2 i-1}^{x} \sigma_{2 i}^{x}+\sigma_{2 i-1}^{y} \sigma_{2 i}^{y}\right)$ competes with the fully polarized triplet components $\{|\uparrow \uparrow\rangle,|\downarrow \downarrow\rangle\}$, which minimize the $\sigma_{2 i-1}^{z} \sigma_{2 i}^{z}$ term on each rung, and a QPT occurs at $\alpha=0.5$ when the rungs do not interact with each other (at $\beta=0$ ). In order to investigate the case of $\beta>0$, we begin with the action of Hamiltonian (1) on the basis states. Operators $\sigma_{i-1}^{z} \sigma_{i+1}^{z}$ give eigenvalues $s_{i-1} s_{i+1}$ for each state (and similar for $\sigma_{2 j-1}^{z} \sigma_{2 j}^{z}$ ), while the products $\sigma_{2 i-1}^{x} \sigma_{2 i}^{x}$ and $\sigma_{2 i-1}^{y} \sigma_{2 i}^{y}$ flip the pairs of spins and may change the sign:

$$
\begin{aligned}
& \sigma_{1}^{x} \sigma_{2}^{x}\left|s_{1}, s_{2}, \cdots, s_{2 N}\right\rangle=\left|-s_{1},-s_{2}, \cdots, s_{2 N}\right\rangle \\
& \sigma_{1}^{y} \sigma_{2}^{y}\left|s_{1}, s_{2}, \cdots, s_{2 N}\right\rangle=-s_{1} s_{2}\left|-s_{1},-s_{2}, \cdots, s_{2 N}\right\rangle .
\end{aligned}
$$

These spin flipping operators act on each rung separately such that spin order inside the rung is conserved. Thus it is convenient to divide the ladder into pairs of spins, antiparallel or parallel, placed on the rungs. Such dimer configurations can be described by vectors $\left|A\left(s_{i}\right)\right\rangle \equiv\left|s_{i},-s_{i}\right\rangle$ for antiparallel and $\left|B\left(s_{i}\right)\right\rangle \equiv\left|s_{i}, s_{i}\right\rangle$ for parallel pairs of spins at rung $i$, respectively.

\subsection{Dimer subspaces}

Now we can label every state of the ladder specifying all dimer types ( $A$ or $B$ ) and all dimer orientations (up or 
down). A crucial observation is that the subspaces with specified dimer types are invariant. For example, a subspace with all dimers parallel is denoted as $B B \ldots B(N$ times $B$ ) and is spanned on $2^{N}$ vectors like

$$
\left|s_{1}^{\prime}, s_{2}^{\prime}, \cdots, s_{N}^{\prime}\right\rangle_{B B \ldots B} \equiv\left|B\left(s_{1}^{\prime}\right), B\left(s_{2}^{\prime}\right), \cdots, B\left(s_{N}^{\prime}\right)\right\rangle
$$

which means a state $\left|s_{1}^{\prime}, s_{1}^{\prime}, s_{2}^{\prime}, s_{2}^{\prime}, \cdots, s_{N}^{\prime}, s_{N}^{\prime}\right\rangle$ in the original notation. A spin excitation $\left|s_{1}^{\prime},-s_{1}^{\prime}, s_{2}^{\prime}, s_{2}^{\prime}, \cdots, s_{N}^{\prime}, s_{N}^{\prime}\right\rangle$ is now labelled by a state in a subspace $A B \ldots B$ :

$$
\left|s_{1}^{\prime}, s_{2}^{\prime}, \cdots, s_{N}^{\prime}\right\rangle_{A B \ldots B} \equiv\left|A\left(s_{1}^{\prime}\right), B\left(s_{2}^{\prime}\right), \cdots, B\left(s_{N}^{\prime}\right)\right\rangle .
$$

Every state of the ladder $\left|s_{1}^{\prime}, s_{2}^{\prime}, \cdots, s_{N}^{\prime}\right\rangle_{d_{1}, d_{2}, \cdots, d_{N}}$, where $d_{i} \in\{A, B\}$ and $i=1,2, \cdots, N$ specifies one subspace. Next we find the form of the Hamiltonian (1) in this dimer representation by introducing new operators $\tau_{i}^{z}$ and $\tau_{i}^{x}$ acting on dimers as if they were single spins:

$$
\begin{aligned}
& \tau_{1}^{z}\left|s_{1}^{\prime}, \cdots, s_{N}^{\prime}\right\rangle_{d_{1}, \cdots, d_{N}}=s_{1}^{\prime}\left|s_{1}^{\prime}, \cdots, s_{N}^{\prime}\right\rangle_{d_{1}, \cdots, d_{N}}, \\
& \tau_{1}^{x}\left|s_{1}^{\prime}, \cdots, s_{N}^{\prime}\right\rangle_{d_{1}, \cdots, d_{N}}=\left|-s_{1}^{\prime}, \cdots, s_{N}^{\prime}\right\rangle_{d_{1}, \cdots, d_{N}} .
\end{aligned}
$$

A similar transformation was introduced for a frustrated spin-1/2 chain by Emery and Noguera [21], who showed that it can be mapped onto an Ising model in a transverse field. Recently this procedure was used to investigate quantum criticality in a two-leg strongly correlated ladder model at quarter filling [22]. The above new operators $\left\{\tau_{1}^{x}, \tau_{1}^{z}\right\}$ will be called pseudospins; using their explicit form and the above $\left|s_{1}^{\prime}, \cdots, s_{N}^{\prime}\right\rangle_{d_{1}, \cdots, d_{N}}$ states, we are able to write the Hamiltonian in any subspace. The simplest result is obtained in the subspaces $A A \ldots A$ and $B B \ldots B$ (here we use periodic boundary condition $\tau_{N+1}^{z} \equiv \tau_{1}^{z}$ ):

$$
\begin{aligned}
& \mathcal{H}_{A A \ldots A}=2 \sum_{i=1}^{N}\left\{K \tau_{i}^{z} \tau_{i+1}^{z}-J_{1} \tau_{i}^{x}\right\}+J_{2} N, \\
& \mathcal{H}_{B B \ldots B}=2 K \sum_{i=1}^{N} \tau_{i}^{z} \tau_{i+1}^{z}-J_{2} N .
\end{aligned}
$$

In the first case (8) one gets the exactly solvable QIM with transverse field [23-25], while in the second case (9) the field is absent and the Hamiltonian is classical. In the subspaces $A B A B \ldots A B$ and $B A B A \ldots B A$ the Hamiltonian is even simpler, with a transverse field acting on pseudospins:

$$
\begin{aligned}
\mathcal{H}_{A B A B \ldots A B} & =-2 J_{1} \sum_{i=1}^{N / 2} \tau_{2 i-1}^{x}, \\
\mathcal{H}_{B A B A \ldots B A} & =-2 J_{1} \sum_{i=1}^{N / 2} \tau_{2 i}^{x} .
\end{aligned}
$$

To get the Hamiltonian in an arbitrary subspace labeled by $\left(d_{1}, d_{2} \cdots, d_{N}\right)$, we introduce new local variables $r_{i}=$ -1 if $d_{i}=A$, and $r_{i}=1$ if $d_{i}=B$. Therefore, we can label each subspace equivalently by a vector $\boldsymbol{r} \equiv\left\{r_{1}, \ldots, r_{N}\right\}$ to obtain a general form of the Hamiltonian

$$
\begin{aligned}
\mathcal{H}_{r_{1}, \cdots, r_{N}}= & \sum_{i=1}^{N}\left\{K\left(1+r_{i} r_{i+1}\right) \tau_{i}^{z} \tau_{i+1}^{z}\right. \\
& \left.-J_{1}\left(1-r_{i}\right) \tau_{i}^{x}-J_{2} r_{i}\right\}
\end{aligned}
$$

where $r_{N+1} \equiv r_{1}$. This result means that in a given subspace $\boldsymbol{r}$ the Hamiltonian indeed reduces to the exactly solvable pseudospin QIM [25]. However, when $r_{i} r_{i+1}=$ -1 , the interaction on the bond $\langle i, i+1\rangle$ is removed, and the chain is fragmented into two parts. Any number of such separated fragments is allowed, so one arrives at a technical difficulty that Ising chains with open ends cannot be solved in general (but see also Sect. 3.2 for more details). Here we recall that for an insulated rung $\langle 2 i-1,2 i\rangle$, i.e. when $r_{i} r_{i+1}=r_{i-1} r_{i}=-1$, the energy of two spins is either $-2 J_{1}+J_{2}$ or $-J_{2}$, depending on whether $r_{i}=-1$ or 1 . The point $J_{1}=J_{2}$ marks a first order QPT where the spin correlation function $\left\langle\sigma_{2 i-1}^{z} \sigma_{2 i}^{z}\right\rangle$ changes discontinuously.

While one cannot find an exact solution for any general Hamiltonian (12) in a general case due to the lack of translational symmetry, fortunately exact solutions can be found in the most symmetric subspaces $A A \ldots A$, $B B \ldots B, A B A B \ldots A B$, and $B A B A \ldots B A$, with their Hamiltonians introduced in equations (8)-(11). We argue that this suffices to determine the ground state of the spin ladder (1). This may be justified by considering the structure of the 1D Ising model (12). One expects that the minimal energy is reached when the maximal number of interacting bonds is present. This suggests that the ground state belongs to the subspace $A A \ldots A$. On the other hand, there is a constant term $J_{2} N$ in equations (8)-(9) which increases the energy in the $A A \ldots A$ subspace but decreases it in the $B B \ldots B$ one. Summarizing, one expects that the ground state belongs to the $B B \ldots B$ subspace for large $J_{2}$, and to the $A A \ldots A$ subspace for large $J_{1}$.

\section{Ground state properties}

\subsection{The quantum Ising model}

To obtain the ground state energy we need to solve the 1D QIM given in equation (8),

$$
\mathcal{H}_{\mathrm{QIM}}=2 \sum_{i=1}^{N}\left\{K \tau_{i}^{z} \tau_{i+1}^{z}-J_{1} \tau_{i}^{x}\right\},
$$

with $N$ even and periodic boundary condition. In eigenbasis of $\tau_{i}^{x}$ we perform Jordan-Wigner transformation replacing pseudospin operators by fermions $c_{i}$ and $c_{i}^{\dagger}$ :

$$
\begin{aligned}
& \tau_{i}^{x}=1-2 c_{i}^{\dagger} c_{i}, \\
& \tau_{i}^{z}=\left(c_{i}+c_{i}^{\dagger}\right) \prod_{j<i}\left(1-2 c_{j}^{\dagger} c_{j}\right) .
\end{aligned}
$$


In terms of new operators $\mathcal{H}_{A A \ldots A}$ of equation (8) is

$$
\begin{aligned}
\mathcal{H}_{\mathrm{QIM}}= & 2 \sum_{i=1}^{N}\left\{K\left(c_{i}^{\dagger}-c_{i}\right)\left(c_{i+1}^{\dagger}+c_{i+1}\right)+2 J_{1} c_{i}^{\dagger} c_{i}\right\} \\
& -2 N J_{1},
\end{aligned}
$$

where $c_{N+1}$ is equal $-c_{1}$ for even and $c_{1}$ for odd total number of $c$-fermions, and subspaces with even/odd number of fermions are invariant. Next step is the Fourier transformation, $c_{j}=(1 / \sqrt{N}) \sum_{k} e^{i j k} c_{k}$, with quasi-momenta $k= \pm(2 l-1) \pi / N(l=1,2, \cdots, N / 2)$ in even subspace, and $k=0, \pi, \pm 2 l \pi / N[l=1,2, \cdots,(N / 2-1)]$ in odd one. We obtain $\mathcal{H}_{\mathrm{QIM}}=\sum_{k} H_{k}-2 N J_{1}$, where

$$
H_{k}=4\left(K \cos k+J_{1}\right) c_{k}^{\dagger} c_{k}+2 L\left(c_{k}^{\dagger} c_{-k}^{\dagger} e^{i k}+H . c .\right)
$$

for $k \neq\{0, \pi\}$. In addition,

$$
H_{0}=4\left(J_{1}+K\right) c_{0}^{\dagger} c_{0}, \quad H_{\pi}=4\left(J_{1}-K\right) c_{\pi}^{\dagger} c_{\pi}
$$

contribute in each odd subspace.

Diagonalization is completed by a Bogoliubov transformation [9], with new operators $\gamma_{k}^{\dagger} \equiv \alpha_{k} c_{k}^{\dagger}+\beta_{k} c_{-k}$ (for $k \neq 0, \pi$, while the operators $c_{0}$ and $c_{\pi}$ are left untransformed), which leads to eigenequations for $\alpha_{k}$ and $\beta_{k}$ obtained from the condition

$$
\left[\mathcal{H}_{\mathrm{QIM}}, \gamma_{k}^{\dagger}\right]=\omega_{k} \gamma_{k}^{\dagger}
$$

We get two eigenvectors $\left(\alpha_{k}, \beta_{k}\right)$ and two corresponding eigenvalues $\omega_{k}= \pm E_{k}$, with

$$
E_{k}=4\left\{K^{2}+J_{1}^{2}+2 K J_{1} \cos k\right\}^{1 / 2} .
$$

These two vectors define new fermion (quasiparticle) operators, called here $\gamma_{k}^{\dagger}$ and $\gamma_{-k}^{\dagger}$ respectively. The Hamiltonian is brought to the diagonal form in both subspaces

$$
\begin{aligned}
\mathcal{H}_{\text {even }}= & \sum_{k>0} E_{k}\left(\gamma_{-k}^{\dagger} \gamma_{-k}+\gamma_{k}^{\dagger} \gamma_{k}-1\right), \\
\mathcal{H}_{\text {odd }}= & \sum_{\pi>k>0} E_{k}\left(\gamma_{-k}^{\dagger} \gamma_{-k}+\gamma_{k}^{\dagger} \gamma_{k}-1\right) \\
& +4\left(J_{1}+K\right) c_{0}^{\dagger} c_{0}+4\left(J_{1}-K\right) c_{\pi}^{\dagger} c_{\pi}-4 J_{1} .
\end{aligned}
$$

Note that the Bogoliubov transformation does not change the total parity of fermions thus only states with even (odd) number of quasiparticles $\gamma_{k}^{\dagger}$ (including $c_{0}^{\dagger}, c_{\pi}^{\dagger}$ ) belong to the spectrum of $\mathcal{H}_{\text {even }}\left(\mathcal{H}_{\text {odd }}\right)$. In general case, the ground state energy depends on the parity of $N$. In the thermodynamic limit $N \rightarrow \infty$ the parity becomes irrelevant, and we can express the energy in the subspace $A A \ldots A$,

$$
E_{A A \ldots A}=-\frac{4 N}{\pi}\left(J_{1}+K\right) E\left[\frac{4 J_{1} K}{\left(J_{1}+K\right)^{2}}\right]+N J_{2},
$$

by a complete elliptic integral $E[x]$ of the second kind.
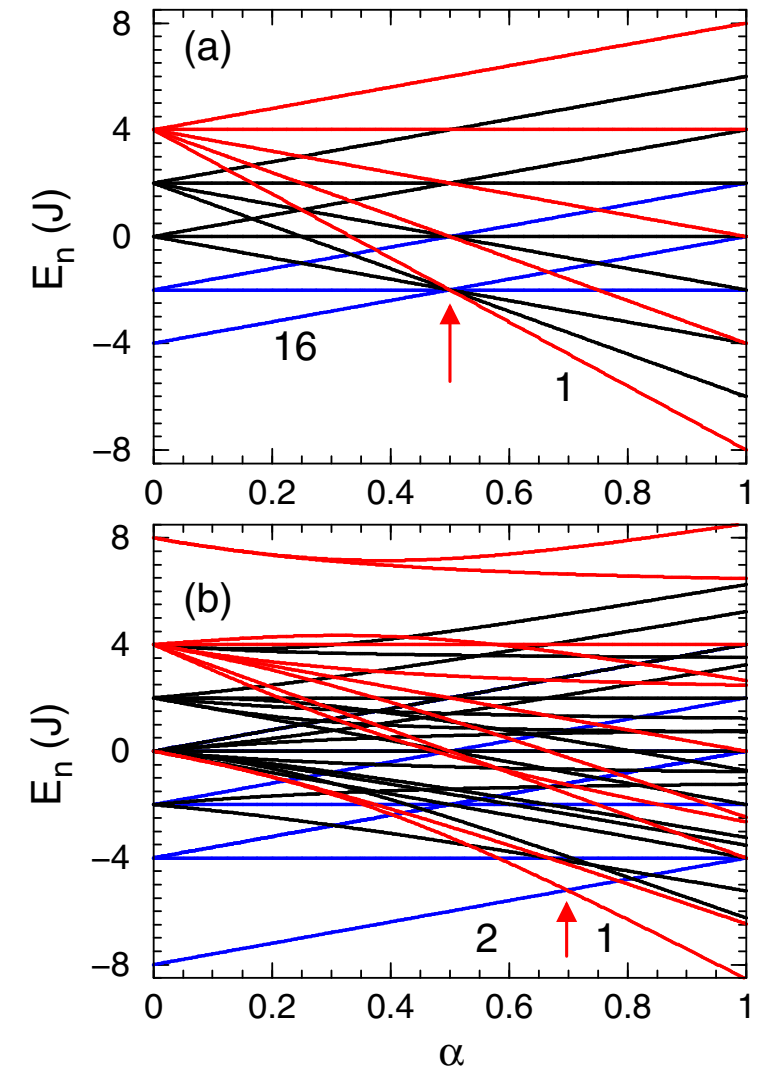

Fig. 2. Eigenenergies $E_{n}$ of the frustrated spin ladder (1) with $N=4$ rungs (shown in figure 1) for increasing $\alpha$, as obtained by exact diagonalization for: (a) $\beta=0$, and (b) $\beta=0.5$. Level crossing at $\alpha_{c}$ (arrows) marks the QPT between the ground states belonging to the $B B \ldots B\left(\alpha<\alpha_{c}\right)$ and $A A \ldots A\left(\alpha>\alpha_{c}\right)$ subspace, with their degeneracies given next to the lines. This transition gives discontinuous changes of the nearest-neighbor spin correlations. The crossing points for excited states at $\alpha=0.5$ in case (b) follow from the degeneracy of $S^{z}= \pm 1$ and $S^{z}=0$ local rung states.

In the $B B \ldots B$ subspace one can easily get the classical value

$$
E_{B B \ldots B}=-2 N K-N J_{2}
$$

from equation (9). Indeed, the classical ground state is found in the $B B \ldots B$ subspace for large $J_{2}$, while for large $J_{1}$ it changes to the ground state of the QIM and belongs to the $A A \ldots A$ subspace, as shown by the analysis of different subspaces for a finite system in Section 3.2.

As an illustrative example, we show the complete spectrum for a finite spin ladder with $N=4$ rungs in Figure 2 . The lowest energies obtained in subspaces $A B A B \ldots A B$ and $B A B A \ldots B A, E_{A B A B \ldots A B}=E_{B A B A \ldots B A}=-N J_{1}$, are higher than either $E_{A A \ldots A}$ or $E_{B B \ldots B}$ for all positive $J_{1}, J_{2}$, and $K$. Increasing value of $K$ moves the transition point between these two subspaces to higher values of $\alpha$, and the quantum regime with $E_{A A \ldots A}<E_{B B \ldots B}$ gradually shrinks, i.e. $\alpha_{c} \rightarrow 1$ for $\beta \rightarrow \infty$, see Figure 3 . Note that although we cannot present rigorous results for a general case of the QIM with some $B$ defects distributed along the $1 \mathrm{D}$ chain starting from the $A A \ldots A$ subspace, 


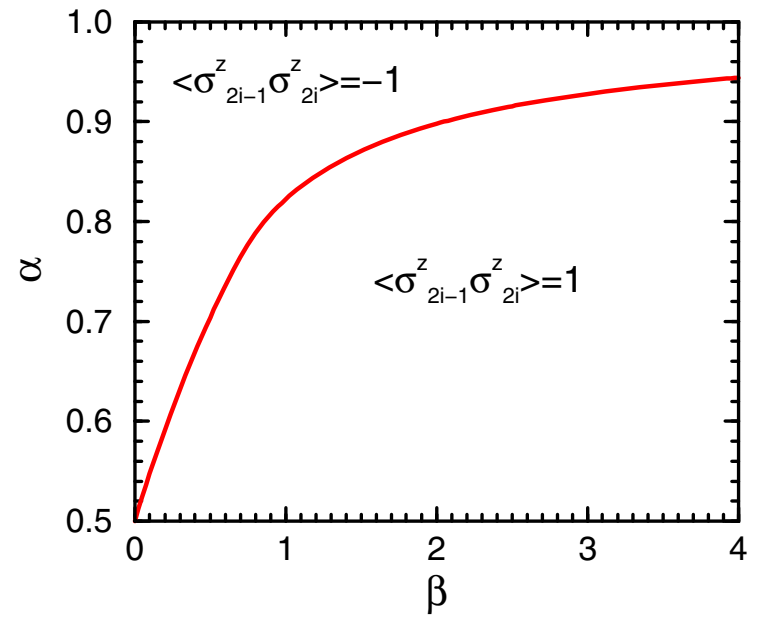

Fig. 3. Phase diagram in the $(\alpha, \beta)$ plane for the spin ladder (1) with frustrated exchange interactions in the thermodynamic limit $N \rightarrow \infty$. The line at $\alpha=\alpha_{c}$ marks the QPT between the classical and quantum regime, with spin correlations on the rungs changing from $\left\langle\sigma_{2 i-1}^{z} \sigma_{2 i}^{z}\right\rangle=1$ to $\left\langle\sigma_{2 i-1}^{z} \sigma_{2 i}^{z}\right\rangle=-1$ at $\alpha_{c}$.

this does not prevent us from finding the ground state and its properties rigorously. The proof that such states have always higher energy than either $E_{A A \ldots A}$ or $E_{B B \ldots B}$ is given in the following subsection.

\subsection{Classification of dimer subspaces}

As explained in Section 2.2, each subspace of the ladder Hamiltonian (1) is defined by a set of indices $\boldsymbol{r} \equiv$ $\left\{r_{1}, r_{2}, \ldots, r_{N}\right\}$. The operator $\tau_{i}^{x}$ occurs in equation (13) only when $r_{i}=-1$, and a quantum component is added. The resulting Hamiltonian is quadratic in Jordan-Wigner fermion operators and may be always diagonalized by a Bogoliubov transformation. This transformation is in general of size $(2 L \times 2 L)$, where its size $1<L \leq N-1$ depends on the subspace $r$. Therefore, for a large number of subspaces one cannot find an exact spectrum in the thermodynamic limit. Of course, there are exceptions. For instance, we have shown above that either the subspace $A A \ldots A$ or $B B \ldots B$ are both exactly solvable. Having calculated energies $E_{A A \ldots A}$ and $E_{B B \ldots B}$ we now have to make sure that these are indeed the minimal energies of the system for $\alpha \geq \alpha_{c}$ and $\alpha<\alpha_{c}$ respectively. If so, then

$$
\Delta E(\alpha) \equiv E_{\star}(\alpha)-E_{\mathrm{gs}}(\alpha)
$$

is positive for all $\alpha$ where $E_{\mathrm{gs}}(\alpha)$ is the ground state energy and $E_{\star}(\alpha)$ is a minimal energy from any subspace excluding $A A \ldots A$ and $B B \ldots B$. In every such subspace the generic Hamiltonian (12) can be written as a sum of commuting operators $\mathcal{H}_{A}\left(a_{i}\right)$ and $\mathcal{H}_{B}\left(b_{i}\right)$ as follows:

$$
\mathcal{H}_{\star}=\sum_{i=1}^{n}\left\{\mathcal{H}_{A}\left(a_{i}\right)+\mathcal{H}_{B}\left(b_{i}\right)\right\},
$$

with

$$
\begin{aligned}
& \mathcal{H}_{A}\left(a_{i}\right)=2 K \sum_{j=1}^{a_{i}-1} \tau_{j}^{z} \tau_{j+1}^{z}-2 J_{1} \sum_{j=1}^{a_{i}} \tau_{j}^{x}+J_{2} a_{i} \\
& \mathcal{H}_{B}\left(b_{i}\right)=2 K \sum_{j=1}^{b_{i}-1} \tau_{j}^{z} \tau_{j+1}^{z}-J_{2} b_{i} .
\end{aligned}
$$

Here $a_{i}\left(b_{i}\right)$ are the lengths of $A(B)$ strings appearing in a subspace label (for example: label $A A B B A A$ has $a_{1}=4$ and $\left.b_{1}=2\right)$. Every label can contain $n(1 \leq n \leq N / 2)$ of $A$ or $B$ strings. Spin operators from different chains are independent even though they are all denoted by $\tau_{j}^{z(x)}$. Defining the minimal energies of $\mathcal{H}_{A}\left(a_{i}\right)$ and $\mathcal{H}_{B}\left(b_{i}\right)$ as $E_{A}\left(a_{i}\right)$ and $E_{B}\left(b_{i}\right)$ one can write $E_{\star}$ as

$$
E_{\star}=\sum_{i=1}^{n}\left\{E_{A}\left(a_{i}\right)+E_{B}\left(b_{i}\right)\right\} .
$$

Next step is to express all appearing energies as follows:

$$
\begin{aligned}
& E_{A}\left(a_{i}\right)=a_{i}\left\{\varepsilon\left(a_{i}\right)+J_{2}\right\}, \\
& E_{A A \ldots A}=N\left(\tilde{\varepsilon}+J_{2}\right)=N \varepsilon_{A},
\end{aligned}
$$

and

$$
\begin{aligned}
E_{B B \ldots B} & =N\left(-2 K-J_{2}\right)=N \varepsilon_{B}, \\
E_{B}\left(b_{i}\right) & =2 K+b_{i} \varepsilon_{B},
\end{aligned}
$$

where the new functions $\varepsilon\left(a_{i}\right), \tilde{\varepsilon}, \varepsilon_{A}$ and $\varepsilon_{B}$ are defined by the right-hand side of equations (30)-(32). The last equation (33) simply follows from equations (32) and (28). Both $E_{A A \ldots A}$ and $E_{B B \ldots B}$ are known from Section 3.1 . Now we can calculate $\Delta E(\alpha)$ putting $E_{\mathrm{gs}}(\alpha)$ equal to $E_{A A \ldots A}(\alpha)$ for $\alpha \geq \alpha_{c}$ and $E_{B B \ldots B}(\alpha)$ for $\alpha<\alpha_{c}$. Using identity $\sum_{i=1}^{n}\left\{a_{i}+b_{i}\right\}=N$ and equations (25), (29)-(33) one finds:

$$
\begin{aligned}
\Delta E\left(\alpha \geq \alpha_{c}\right)= & \sum_{i=1}^{n}\left\{a_{i}\left[\varepsilon\left(a_{i}\right)-\tilde{\varepsilon}\right]+b_{i}\left(\varepsilon_{B}-\varepsilon_{A}\right)\right\} \\
& +2 K n
\end{aligned}
$$

and

$$
\begin{aligned}
\Delta E\left(\alpha<\alpha_{c}\right)= & \sum_{i=1}^{n}\left\{a_{i}\left[\varepsilon\left(a_{i}\right)-\tilde{\varepsilon}\right]+b_{i}\left(\varepsilon_{A}-\varepsilon_{B}\right)\right\} \\
& +2 K n .
\end{aligned}
$$

In both equations the second term under the sum is positive. The first term cannot be calculated without fixing $a_{i}$ because it contains $\varepsilon\left(a_{i}\right)$ which is the ground state energy per site of a QIM chain with loose ends. Similarly, $\tilde{\varepsilon}$ is the ground state energy per site of the same QIM chain but with a closing bond and of the length $N>a_{i}$. This suggests that $\left\{\varepsilon\left(a_{i}\right)-\tilde{\varepsilon}\right\}$ should be always positive but let us examine this on a finite chain. Luckily, $\varepsilon\left(a_{i}\right)$ can 


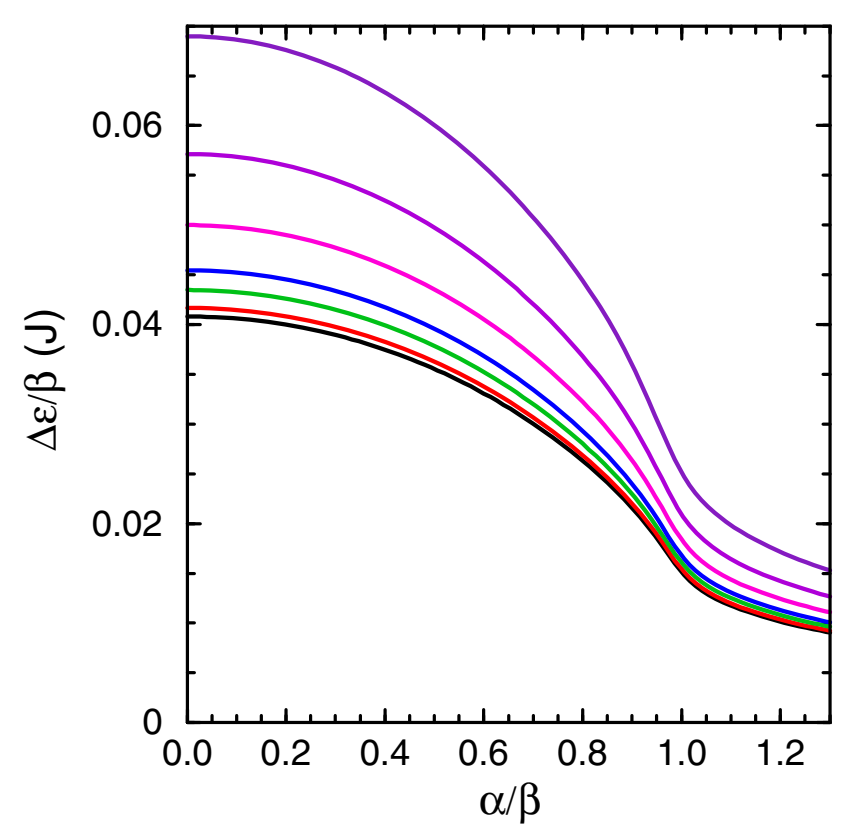

Fig. 4. Energy difference $\Delta \varepsilon$ as obtained for the chain of length $N=50$, with the length of $A$ strings being $a_{i}=$ $49,48,46,44,40,35,29$, from bottom to top.

be obtained numerically as a function of $\alpha$ and $\beta$ even for rather big $a_{i}$. This can be done by performing a Bogoliubov transformation of Jordan-Wigner fermion operators and finding eigenvalues of $\left(2 a_{i} \times 2 a_{i}\right)$ matrix [25]. The energy difference,

$$
\Delta \varepsilon \equiv \varepsilon\left(a_{i}\right)-\tilde{\varepsilon},
$$

normalized by $\beta$ is shown in a Figure 4 as a function of increasing $\alpha / \beta$ for $N=50$ and $a_{i}=49,48,46,44,40,35,29$. Each curve starts at a certain positive value for $\alpha / \beta=0$ and decreases asymptotically to zero which agrees with a physical interpretation of $\Delta \varepsilon(36)$.

We have proven that the ground state of the Hamiltonian (1) can be either in $A A \ldots A$ or $B B \ldots B$ subspace. This means that at the transition point $\alpha=\alpha_{c}$ (Fig. 3) all the triplets on the rungs disappear simultaneously, without passing through any intermediate state with fractional number of triplets per rung (which one might expect for finite interactions along the legs).

\subsection{Correlation functions}

The spin correlations in the ground state depend on the interaction parameters $\left\{J_{1}, J_{2}, K\right\}$. The nearest-neighbor correlations change discontinuously at $\alpha=\alpha_{c}$ (see Fig. 5), where the lowest energy levels $E_{A A \ldots A}(23)$ and $E_{B B \ldots B}$ (24) cross each other and the nature of the ground state changes. The $B B \ldots B$ subspace is classical and the only non-zero correlations are those involving diagonal operators $\sigma_{i}^{z}$ - one finds FM correlations $\left\langle\sigma_{2 i-1}^{z} \sigma_{2 i}^{z}\right\rangle_{B B \ldots B}=1$ on the rungs, and AF ones $\left\langle\sigma_{i-1}^{z} \sigma_{i+1}^{z}\right\rangle_{B B \ldots B}=-1$ between
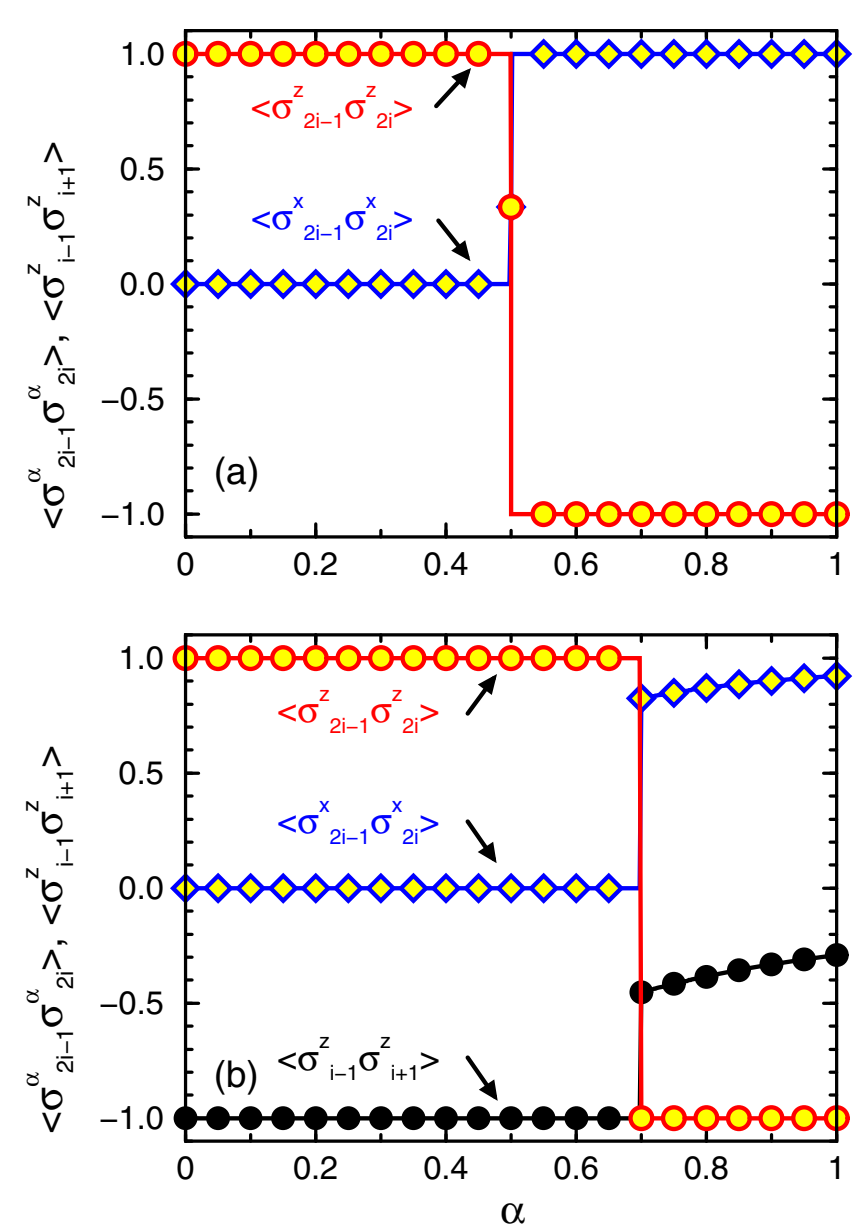

Fig. 5. Intersite spin correlations on the rungs $\langle 2 i-1,2 i\rangle$ and on the bonds $\langle i-1, i+1\rangle$ along the legs obtained for the spin ladder equation (1) in the thermodynamic limit $N \rightarrow \infty$ for increasing $\alpha$, and for: (a) $\beta=0$, (b) $\beta=0.5$. A transition at $\alpha_{c}$ separates two types of pseudo-order on the rungs: (i) $\left\langle\sigma_{2 i-1}^{z} \sigma_{2 i}^{z}\right\rangle=1$ and $\left\langle\sigma_{2 i-1}^{x} \sigma_{2 i}^{x}\right\rangle=0$ for $\alpha<\alpha_{c}$, and $(i i)$ $\left\langle\sigma_{2 i-1}^{z} \sigma_{2 i}^{z}\right\rangle=-1$ and $\left\langle\sigma_{2 i-1}^{x} \sigma_{2 i}^{x}\right\rangle>0$ for $\alpha>\alpha_{c}$. This transition is accompanied by a discontinuous change of intersite spin correlations $\left\langle\sigma_{i-1}^{z} \sigma_{i+1}^{z}\right\rangle$ along the legs in case (b).

them. In contrast, the $A A \ldots A$ subspace is quantum, and the $\left\langle\sigma_{i-1}^{z} \sigma_{i+1}^{z}\right\rangle_{A A \ldots A}$ correlations are those of the QIM [9].

At $K=0(\beta=0)$ the correlation function $\left\langle\sigma_{2 i-1}^{z} \sigma_{2 i}^{z}\right\rangle$ changes discontinuously from 1 to -1 at $\alpha_{c}=0.5$, and simultaneously $\left\langle\sigma_{2 i-1}^{x} \sigma_{2 i}^{x}\right\rangle$ switches from 0 to 1 [Fig. 5a]. The change from FM to AFM local correlation on the rungs is at first instance surprising and occurs in spite of still FM interaction $\propto-J_{1} \sigma_{2 i-1}^{z} \sigma_{2 i}^{z}$ between the $z$-th spin components - simply the system gains more energy from the transverse $\propto\left(\sigma_{2 i-1}^{x} \sigma_{2 i}^{x}+\sigma_{2 i-1}^{y} \sigma_{2 i}^{y}\right)$ terms. At the transition point $\alpha=0.5$ one finds a high degeneracy (e.g. $d=81$ for $N=4$ ) of the ground state, and all three triplet components on each rung are equivalent, consequently the spin correlations are: $\left\langle\sigma_{2 i-1}^{z} \sigma_{2 i}^{z}\right\rangle=\left\langle\sigma_{2 i-1}^{x} \sigma_{2 i}^{x}\right\rangle=1 / 3$.

The nearest neighbor correlations exhibit similar discontinuous changes at the QPT (Fig. 3) when $K$ is finite $(\beta>0)$. While the spin correlations in Figure $5 \mathrm{~b}$ 
are again classical for $\alpha<\alpha_{c}$ (i.e., $\left\langle\sigma_{i-1}^{z} \sigma_{i+1}^{z}\right\rangle=-1$ and $\left.\left\langle\sigma_{2 i-1}^{z} \sigma_{2 i}^{z}\right\rangle=1\right)$, they are nontrivial for $\alpha>\alpha_{c}$ and can be obtained from the energy (23) by taking its partial derivatives:

$$
\begin{aligned}
\left\langle\sigma_{2 i-1}^{z} \sigma_{2 i+1}^{z}\right\rangle_{A A \ldots A} & =\left.\frac{1}{2 N}\left(\frac{\partial E_{A A \ldots A}}{\partial K}\right)\right|_{K=\beta J} \\
\left\langle\sigma_{2 i-1}^{x} \sigma_{2 i}^{x}\right\rangle_{A A \ldots A} & =-\left.\frac{1}{2 N}\left(\frac{\partial E_{A A \ldots A}}{\partial J_{1}}\right)\right|_{J_{1}=\alpha J}
\end{aligned}
$$

Note that the spin correlations on the rungs,

$$
\begin{aligned}
& \left\langle\sigma_{2 i-1}^{z} \sigma_{2 i}^{z}\right\rangle=r_{i}, \\
& \left\langle\sigma_{2 i-1}^{x} \sigma_{2 i}^{x}\right\rangle=\left\langle\tau_{i}^{x}\right\rangle,
\end{aligned}
$$

follow in general from the QIM (13); the latter one is nontrivial only in the quantum regime for $\alpha>\alpha_{c}$ (the same result is obtained for $\left.\left\langle\sigma_{2 i-1}^{y} \sigma_{2 i}^{y}\right\rangle\right)$. In this subspace one finds again that the transverse spin interactions $\propto$ $\left(\sigma_{2 i-1}^{x} \sigma_{2 i}^{x}+\sigma_{2 i-1}^{y} \sigma_{2 i}^{y}\right)$ dominate and $\sigma_{2 i-1}^{z} \sigma_{2 i}^{z}$ terms are frustrated when AFM correlations, $\left\langle\sigma_{2 i-1}^{z} \sigma_{2 i}^{z}\right\rangle_{A A \ldots A}=-1$, occur on the rungs. Simultaneously the AFM correlations $\left\langle\sigma_{i-1}^{z} \sigma_{i+1}^{z}\right\rangle$ weaken along the ladder, as shown in Figure $5 \mathrm{~b}$.

Finally, the long-range spin correlations along the ladder legs are:

$$
\begin{aligned}
& \left\langle\sigma_{2 i+a}^{z} \sigma_{2 j+b}^{z}\right\rangle=r_{i}^{a+1} r_{j}^{b+1}\left\langle\tau_{i}^{z} \tau_{j}^{z}\right\rangle, \\
& \left\langle\sigma_{2 i+a}^{x} \sigma_{2 j+b}^{x}\right\rangle=0
\end{aligned}
$$

with $a, b=0,1$. The first correlation function follows from the spin correlations $\left\langle\tau_{i}^{z} \tau_{j}^{z}\right\rangle$ in the QIM given by equation (13). The second one vanishes for all $\{i, j\}$ as the operator $\sigma_{2 i+a}^{x} \sigma_{2 j+b}^{x}$ creates an excitation which belongs to a subspace which is orthogonal to the one containing the ground state.

\section{Spin excitations}

Although not all excited states can be obtained explicitly for any size of the system, certain general statements are possible by analyzing numerical results for sufficiently large ladders. In contrast to magnon excitations which occur when a a single spin is reversed, we consider here all possible excited states. Therefore, one can expect $a$ priori two types of excitations: $(i)$ the ones within the ground state subspace, and $(i i)$ the ones with an excited state belonging to a different dimer subspace. First ones correspond to operations that do not break the symmetry between two legs of the ladder, for example, flipping two spins on a single rung. Excitations of the second kind break this symmetry, they flip only one out of two spins belonging to a rung which changes the label of a subspace. Numerical results for finite chains prove that the lowest energy excitations of such type lead to $A B B \ldots B$ and $B A A \ldots A$ subspaces for $\alpha<\alpha_{c}$ and $\alpha>\alpha_{c}$ respectively. Apart from that, we still have excitations of the

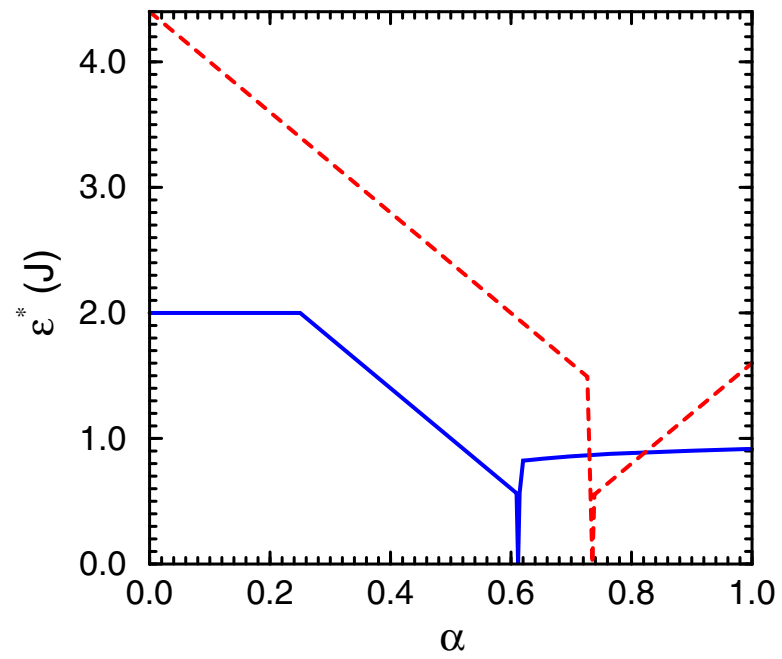

Fig. 6. Excitation energy $\varepsilon^{\star}(\alpha)$ for increasing $\alpha$ obtained for a system of $N=50$ rungs with $\beta=0.25$ (solid line) and $\beta=0.6$ (dashed line). Excited state changes for each section of the curves. For $\beta=0.25$ excited state belongs to $B B \ldots B$ subspace as long as $\alpha<0.5-\beta$ with energy gap $\varepsilon^{\star}(\alpha)=$ $8 K$. Then the gap decreases linearly for excitations leading to $A B B \ldots B$ subspace. The decrease becomes abrupt in vicinity of the QCP (where the new ground state is formed) and the energy gap $\varepsilon^{\star}$ vanishes at $\alpha=\alpha_{c}$. Excitations in the quantum phase out of the critical region involve $B A A \ldots A$ subspace and depend weakly on $\alpha$. In contrast, for $\beta=0.6$ one finds: $(i)$ no $B B \ldots B$ excitations for low $\alpha$, and (ii) Bogliubov excitations within $A A \ldots A$ subspace in a quantum phase with a linear growth of the energy gap.

first kind which are Ising excitations in the classical phase and Bogoliubov excitations in the quantum one. To get the energy gap one has to decide for every $\beta$ and $\alpha$ which excitation has the lowest energy.

As an example, we show in Figure 6 the energy gap (excitation energy) $\varepsilon^{\star}(\alpha)$ for increasing $\alpha$ in case of $\beta=0.25$ and $\beta=0.6$ for a ladder consisting of 50 rungs. Two characteristic features of these plots are: $(i)$ close to the QCP the gap drops and grows back in relatively high rate, and (ii) for higher $\beta$ the curve becomes simpler. The first feature is due to the fact that excitations switch between two possible ground states in the critical region excitations, with energy depending very strongly on $\alpha$. The second one can be explained by the fact that for high $\beta(\beta>0.5)$ the classical phase is completely dominated by an excitation leading to $A B B \ldots B$ subspace, while in the quantum case the excitation is made within its ground subspace. This latter excitation is collective one and is performed by a creation of a single Bogoliubov quasiparticle with momentum $k=\pi$ (which is equivalent to switching between symmetric and antisymmetric linear combination of the two possible Néel configurations in case of zero transverse field). This result confirms the quantum nature of the ground state for $\alpha>\alpha_{c}$.

There are still some subtleties concerning the problem of excited states that will not be discussed here. First of all, one can find such a value of $\beta$ between 0.25 and 0.5 
that in a quantum phase one gets excitations within the ground subspace for $\alpha<\alpha^{\prime}$ and excitations leading out of this subspace for $\alpha>\alpha^{\prime}$ (where $\alpha_{c}<\alpha^{\prime}<1$ ). This results from a fusion of the two cases displayed in Figure 6. The second subtlety arises in the thermodynamic limit $N \rightarrow \infty$. In such a case the state with a single Bogoliubov quasiparticle with momentum $k=\pi$ has the same energy as the Bogoliubov vacuum as long as $\alpha \leq \beta$. Therefore, if the energy splitting appears after passing through the QCP, the first excited state would be always in the $B A A \ldots A$ subspace for $\alpha<\beta$ and outside the critical region. Such a complex variation of the nature and energy of the lowest possible excitation, depending strongly on $\beta$, shows that even infinitesimal interaction between the rungs (i.e., along ladder legs) makes our ladder far more interesting system than a set of noninteracting rungs which also exhibits a QPT.

\section{Discussion and summary}

We have verified that frustrated interactions in the spin ladder (1) do not generate any QPT in several situations, but instead the ground state changes gradually and the intersite spin correlation functions change smoothly with varying interaction parameters. Such a behavior occurs, for example, when FM Ising interactions along the ladder legs coexist with AFM Heisenberg interactions on the rungs. On the contrary, we have shown that a first order QPT occurs when the interactions lead to competition between the spin $s^{z}= \pm 1$ and $s^{z}=0$ triplet component on each rung.

The present method of solving the problem in different dimer subspaces separately elucidates the origin of the quantum phase transition found in the present spin ladder model. We argue that this approach could help to find exact solutions for a class of quasi-1D models with frustrated spin interactions, in cases when spin interactions along the ladder legs are Ising-like. The latter condition is essential for constructing an exact solution.

Summarizing, the spin ladder studied in this paper exhibits a first order QPT from a classical to a quantum ground state which occurs due to the level crossing. It leads to a discontinuous change of intersite spin correlations when: $(i)$ the interactions on the rungs are anisotropic and describe competition between the components of local triplets, and ( $i i)$ the interactions along the ladder legs are Ising-like. Fortunately, the subspaces which are relevant for the QPT in the frustrated spin ladder (1) considered here can be analyzed rigorously by mapping the ladder to the quantum Ising model which determines both the ground state energy and spin correlation functions. The analysis of the $B B B \cdots B A B \cdots B$ and $A A A \cdots A B A \cdots A$ subspaces performed for arbitrary $\alpha$ and $\beta$ in a finite systems demonstrates that no fluctuations will spoil the first order transition which occurs in the spin ladder (1) when $\alpha$ increases.
We acknowledge support by the Foundation for Polish Science (FNP) and by the Polish Ministry of Science and Higher Education under Project No. N202 068 32/1481.

\section{References}

1. E. Dagotto, Rep. Prog. Phys. 62, 1525 (1999)

2. S. Gopalan, T.M. Rice, M. Sigrist, Phys. Rev. B 49, 8901 (1994)

3. P. Horsch, F. Mack, Eur. Phys. J. B 5, 367 (1998)

4. S. Trebst, H. Monien, C.J. Hamer, Z. Weihong, R.R.P. Singh, Phys. Rev. Lett. 85, 4373 (2000)

5. C. Knetter, K.P. Schmidt, M. Grüninger, G.S. Uhrig, Phys. Rev. Lett. 87, 167204 (2001)

6. S. Notbohm, P. Ribeiro, B. Lake, D.A. Tennant, K.P. Schmidt, G.S. Uhrig, C. Hess, R. Klingeler, G. Behr, B. Büchner, M. Reehuis, R.I. Bewley, C.D. Frost, P. Manuel, R.S. Eccleston, Phys. Rev. Lett. 98, 027403 (2007)

7. K. Penc, J.-B. Fouet, S. Miyahara, O. Tchernyshyov, F. Mila, Phys. Rev. Lett. 99, 117201 (2007)

8. L. Longa, A.M. Oleś, J. Phys. A 13, 1031 (1980)

9. W. Brzezicki, J. Dziarmaga, A.M. Oleś, Phys. Rev. B 75, 134415 (2007)

10. L.F. Feiner, A.M. Oleś, J. Zaanen, Phys. Rev. Lett. 78, 2799 (1997)

11. S.D. Matteo, G. Jackeli, C. Lacroix, N.B. Perkins, Phys. Rev. Lett. 93, 077208 (2004); S.D. Matteo, G. Jackeli, N.B. Perkins, Phys. Rev. B 72, 024431 (2005); F. Vernay, K. Penc, P. Fazekas, F. Mila, Phys. Rev. B 70, 014428 (2004); F. Vernay, A. Ralko, F. Becca, F. Mila, Phys. Rev. B 74, 054402 (2006); G. Jackeli, D.I. Khomskii, Phys. Rev. Lett. 100, 147203 (2008)

12. G. Khaliullin, S. Maekawa, Phys. Rev. Lett. 85, 3950 (2000); L.F. Feiner, A.M. Oleś, Phys. Rev. B 71, 144422 (2005)

13. A.M. Oleś, P. Horsch, L.F. Feiner, G. Khaliullin, Phys. Rev. Lett. 96, 147205 (2006)

14. J. Zaanen, A.M. Oleś, P. Horsch, Phys. Rev. B 46, 5798 (1992); J. van den Brink, P. Horsch, A.M. Oleś, Phys. Rev. Lett. 85, 5174 (2000); M. Daghofer, K. Wohlfeld, A.M. Oleś, E. Arrigoni, P. Horsch, Phys. Rev. Lett. 100, 066403 (2008)

15. J. van den Brink, New J. Phys. 6, 201 (2004)

16. D.I. Khomskii, M.V. Mostovoy, J. Phys. A 36, 9197 (2003)

17. J. Dorier, F. Becca, F. Mila, Phys. Rev. B 72, 024448 (2005)

18. S. Wenzel, W. Janke, Phys. Rev. B 78, 064402 (2008)

19. J.H.H. Perk, H.W. Capel, M.J. Zuilhof, T.J. Siskens, Physica A 81, 319 (1975)

20. Generic orbital superexchange interactions in Mott insulators are AFM, see K.I. Kugel, D.I. Khomskii, Sov. Phys. Usp. 25, 231 (1982); the present model gives equivalent results for the FM interactions along the ladder legs

21. V.J. Emery, C. Noguera, Phys. Rev. Lett. 60, 631 (1988)

22. S. Lal, M.S. Laad, J. Phys.: Condens. Matter 20, 235213 (2008)

23. E. Lieb, T. Schultz, D. Mattis, Ann. Phys. 16, 407 (1961); S. Katsura, Phys. Rev. 127, 1508 (1962)

24. J. Dziarmaga, Phys. Rev. Lett. 95, 245701 (2005)

25. D.C. Mattis, The Theory of Magnetism Made Simple (World Scientific, Singapore, 2006) 